\title{
On the road again: Wie kann die Arbeitsgestaltung zur Arbeitsfreude bei mobiler Arbeit beitragen?
}

\author{
Kerstin Rieder $\cdot$ Sylvia Kraus $\cdot$ Gerlinde Vogl
}

Online publiziert: 21. Januar 2020

(C) Der/die Autor(en) 2020

Zusammenfassung In dem Beitrag geht es um die Frage, welche Merkmale berufsbedingt mobiler Arbeit einen Zusammenhang mit Arbeitsfreude - als Indikator von intrinsischer Motivation - haben. In einer Studie wurden 407 mobil Beschäftigte schriftlich und 59 mittels Intensivinterviews befragt. Die Ergebnisse zeigen, dass sowohl allgemeine arbeitsbezogene Ressourcen (Handlungsspielraum, Vielseitigkeit der Arbeitsinhalte, Führungsqualität) als auch mobilitätsbezogene Ressourcen (technisch-organisatorische Unterstützung unterwegs) die Arbeitsfreude stärken. In der Diskussion werden Empfehlungen für die Unternehmenspraxis abgeleitet.

Schlüsselwörter Mobile Arbeit · Arbeitsfreude · betriebliche Gesundheitsförderung

Prof. Dr. K. Rieder $(\bowtie) \cdot$ S. Kraus, M. Sc.

Gesundheitsmanagement, Hochschule Aalen, Beethovenstr. 1, 73430 Aalen, Deutschland

E-Mail: kerstin.rieder@hs-aalen.de

S. Kraus, M. Sc.

E-Mail: sylvia.kraus@hs-aalen.de

Dr. G. Vogl

Lehrstuhl für Betriebswirtschaft, Universität Oldenburg, Ammerländer

Heerstraße 114-118, 26129 Oldenburg, Deutschland

E-Mail: Gerlinde.Vog1@ sozialforschung.org 


\section{On the road again: How do working conditions contribute to the joy at mobile work?}

Abstract Aim of the study was to analyze working conditions in the context of mobile work that contribute to joy at work as an indicator of intrinsic motivation. In a study, a total of 407 mobile employees completed a questionnaire, and 59 took part in intensive interviews. The results indicate that general work-related issues (control, task variety and leadership quality) as well as mobility-related resources (technical and organizational support "on the way") enhance joy at work. On this basis, recommendations for the design of mobile work in enterprises are presented.

Keywords Mobile work · joy at work · occupational health

\section{Mobile Arbeit - Stressor oder Abwechslung vom Alltag?}

Digitalisierung und Globalisierung prägen die Arbeitswelt zunehmend (Roth 2017). Damit gewinnen flexible und mobile Arbeit an Bedeutung. Gearbeitet wird beim Kunden, im Café, unterwegs oder im Shared-Office. Von berufsbedingter Mobilität wird gesprochen, wenn diese aus den Anforderungen der Arbeit hervorgeht, z. B. bei Vor-Ort Arbeit, Dienstreisen oder Entsendungen (Ducki und Nguyen 2016). Gegenstand des vorliegenden Beitrags ist die berufsbedingte Mobilität in Form von Dienstreisen.

Nach Angaben des Verbands deutsches Reisemanagement (2019) fanden im letzten Jahr 189,6 Mio. Geschäftsreisen statt. Die Anzahl der Dienstreisen steigt nicht nur in Deutschland, sondern auch weltweit jährlich an. Kesselring und Vogl (2010) sprechen demgemäß von einer „Normalisierung der Mobilitätsanforderungen“. Denn auch viele bislang stationäre Berufe wie kaufmännische Angestellte, Facharbeiter, Techniker oder Pflegeberufe werden zunehmend mobil. Berufliche Mobilität ist demnach heute keine Frage der Qualifikation oder Hierarchie mehr (Nies et al. 2017).

Mit der Verbreitung berufsbedingter Mobilität rückt die Frage in den Fokus, ob diese eher als Stressor oder als Abwechslung vom Alltag wahrgenommen wird. Hier setzt das Forschungsprojekt Präventionsorientierte Gestaltung mobiler Arbeit an (Breisig und Vogl 2019). Das vom Bundesministerium für Bildung und Forschung geförderte Verbundprojekt verfolgte das Ziel, Bedingungen in der Arbeit zu identifizieren, die dazu beitragen, dass mobile Arbeit positiv erlebt wird und persönliches Wachstum in der Arbeit ermöglicht.

\section{Besonderheiten der berufsbedingten Mobilität}

Trotz der wachsenden Bedeutung mobiler Arbeit in der Wirtschaft ist noch wenig darüber bekannt, mit welchen Ressourcen bzw. Positivmerkmalen diese einhergeht. Etwas mehr, allerdings mit Sicherheit noch nicht ausreichend, wurde bisher zu Belastungsfaktoren der mobilen Arbeit geforscht. 
Charakteristisch für berufsbedingte Mobilität sind zum einen allgemeine Belastungen wie Zeitdruck, hohe Arbeitsintensität und häufige Unterbrechungen (Ducki und Nguyen 2016). Dies zeigen etwa Ergebnisse des Projekts prentimo (Kraus und Rieder 2019): In einer querschnittlichen quantitativen Befragung stellten sich insbesondere quantitative (zu viel Arbeit) und qualitative Belastungen (zu schwierige Arbeit) als Prädiktoren für psychische Beanspruchung bei berufsbedingter Mobilität heraus. Ähnliche Ergebnisse erbrachte die Studie zum Belastungserleben bei mobiler Arbeit von Vogl et al. (2014). Belastend erlebt wurde unter anderem quantitative Überforderung.

Darüber hinaus spielen mobilitätsspezifische Belastungen eine bedeutende Rolle: Mobile Mitarbeitende sind oft als „Einzelkämpfer“ unterwegs und erfahren wenig direkte soziale Unterstützung. Abwesenheitsbedingt sind sie von Informationen und Mitsprachemöglichkeiten im Unternehmen vielfach ausgeschlossen. Hinzu kommen lange Fahr- und Abwesenheitszeiten sowie verkehrsbedingte Risiken (u. a. Ducki und Nguyen 2016; Hupfeld et al. 2013; Kraus und Rieder 2019; Mäkelä und Kinnunen 2016; Vogl et al. 2014). Eine erhöhte Beanspruchung zeigt sich auch bei hoher Frequenz der Mobilität (Espino et al. 2002; Nicholas und McDowall 2012). Finden weniger Einsätze pro Jahr statt, zeigt sich kein Zusammenhang mit psychischer Beanspruchung (Jensen 2014). Offen ist in dieser Thematik aktuell noch die Rolle der ständigen Erreichbarkeit, die typisch für mobile Arbeit ist (u. a. Pangert et al. 2017; Rau und Göllner 2019; Vogl et al. 2014). Einerseits stehen die Beschäftigten unter Druck, für gewisse Belange der Arbeit erreichbar sein zu müssen, andererseits entsteht durch den Kontakt aber auch eine gewisse Zugehörigkeit zum Unternehmen und dem Team.

Es sind aber auch positive Aspekte bekannt: Was bei einigen stationären Tätigkeiten demotiviert, die immer gleiche Routine, die Standards oder detailgenauen Vorgaben von Vorgesetzten, spielt bei mobiler Arbeit kaum eine Rolle. In Untersuchungen zeigten sich daher Handlungsspielraum, Vielseitigkeit der Arbeitsinhalte, Unternehmensidentifikation sowie Steuerung über Ziele als positive Prädiktoren für das allgemeine Wohlbefinden bei mobilen Beschäftigten (u.a. Ducki und Nguyen 2016; Hupfeld et al. 2013; Lüdemann 2016).

Darüber hinaus gibt es Studien, die zeigen, dass die berufliche Mobilität als solche entlastend sein kann. Sie kann offenbar beim Detachment von Stressoren im Alltag helfen und so entlastend und befreiend für die Beschäftigten sein (Westman und Etzion 2002). Nach Westman et al. (2009) ist dabei insbesondere hilfreich, wenn Beschäftigte eine gewisse Kontrolle über die Reiseplanung haben. Dies bestätigen auch die Studie von Vogl et al. (2014) sowie aktuelle Untersuchungen aus prentimo (Kraus und Rieder 2019). Eine entsprechende Vorbereitung der Reise kann dazu ebenfalls beitragen (Niessen et al. 2018). Darüber hinaus hat sich gezeigt, dass sich die Unterstützung des Unternehmens, z. B. in Form von Erholungszeiten nach den Reisen und Sicherheitsvorkehrungen für die Reise, positiv auf die Zufriedenheit der Beschäftigten mit Dienstreisen auswirkt (Mäkelä und Kinnunen 2016). Somit scheint berufsbedingte Mobilität zwei Seiten zu haben, hohe Belastungen einerseits und hohe Ressourcen andererseits. 


\section{Arbeitsfreude bei berufsbedingter Mobilität}

Die Beschreibung von zwei Seiten der Arbeit, von Negativ- und Positivmerkmalen, findet sich in den meisten allgemeinen arbeitspsychologischen Modellen (Ulich und Wülser 2018). Exemplarisch kann hier auf das Job Demands-Resources Modell von Bakker und Demerouti $(2014,2017)$ eingegangen werden. Dabei stellen Anforderungen (vergleichbar mit „Belastungen“ in anderen Modellen) alle diejenigen physischen, sozialen oder organisationalen Aspekte der Arbeit dar, die psychische oder physische Anstrengungen erfordern und daher mit psychischen oder physischen Kosten verbunden sind. Demgegenüber sind Ressourcen der Arbeit diejenigen Aspekte, die entweder einen puffernden Effekt auf die negativen Auswirkungen von Anforderungen haben oder förderlich für die individuelle Zielerreichung sind. Sie sind somit auch mit motivationalen Prozessen und mit persönlicher Weiterentwicklung assoziiert (ebd.). Exemplarische Ressourcen sind Handlungsspielraum, Sinnhaftigkeit, soziale Unterstützung, Anforderungsvielfalt sowie Leistungsrückmeldung und Weiterentwicklungsmöglichkeiten im Unternehmen (Bakker und Demerouti 2017; Fried und Ferris 1987; Hackman und Oldham 1980).

Nach Bakker und Demerouti (2008) können aus den vorhandenen Ressourcen zwei Arten der Motivation resultieren: Zum einen sind Ressourcen hilfreich zur Erreichung der Arbeitsziele und resultieren somit in extrinsischer Motivation. Die Ressourcen können allerdings auch mit intrinsischer Motivation assoziiert werden. Dies ist dann der Fall, wenn sie mit Lernen, Entwicklung und Wachstum der Beschäftigten verbunden sind (ebd.). Motivierend sind nicht äußere (externe) Anreize, sondern die Arbeit selbst (Fried und Ferris 1987; Gagné und Deci 2005; Hackman und Oldham 1980). Demnach kann Arbeitsfreude als Indikator für intrinsische Motivation angesehen werden.

Arbeitsfreude lässt sich als „Ausmaß von positiven Emotionen in Bezug auf die eigene Arbeit“ (Ducki 2000, S. 134) verstehen. Freude wird in engem Zusammenhang mit Stolz gesehen und verortet bei Tätigkeiten, die große Spielräume beinhalten, hohe Kompetenz erfordern und Produkte hoher Qualität zum Ergebnis haben (Bornheim-Gallemeister 2013). Eine Abgrenzung von anderen positiven arbeitsbezogenen Emotionen und eine genauere wissenschaftliche Beschreibung der Arbeitsfreude erweist sich als herausfordernd, so dass hierzu mitunter auf die Lyrik verwiesen wird (Meller und Ducki 2002). Arbeitsfreude kann prozessbezogen, ereignisbezogen oder ergebnisbezogen auftreten, wobei vielfach der prozessbezogene Charakter der Freude betont wird (Bornheim-Gallemeister 2013).

Bislang liegen kaum Studien vor, die sich mit dem motivationalen Aspekt typischer Ressourcen mobiler Arbeit befassen. Mäkelä und Kinnunen (2016) untersuchten den Zusammenhang von organisationaler Unterstützung mit der Schaffenskraft (vigor) der Beschäftigten und der Zufriedenheit mit der mobilen Tätigkeit und fanden positive Effekte. Eine Studie von Niessen et al. (2018) zeigt, dass die Kumulierung von Ressourcen vor einer Reise unter anderem mit höherem Arbeitsengagement einhergeht. Darüber hinaus ist die entsprechende Studienlage spärlich. 


\section{Fragestellungen und Hypothesen}

Die vorliegende Arbeit soll dazu beitragen, die Erkenntnisse zu arbeitsbezogenen Ressourcen bei berufsbedingter Mobilität zu vertiefen. Es wird untersucht, welchen Beitrag diese zum Entstehen von Arbeitsfreude leisten. Dabei wird unterschieden zwischen allgemeinen Arbeitsressourcen - z. B. Handlungsspielraum, Vielseitigkeit oder Führungsqualität (Bakker und Demerouti 2017; Hupfeld et al. 2013) - und mobilitätsbezogenen Arbeitsressourcen - z. B. mobilitätsbezogener Planungsspielraum sowie mobilitätsbezogene Unterstützung (Hupfeld et al. 2013; Mäkelä und Kinnunen 2016; Müller et al. 2017). Die erste Hypothese lautet:

Hypothese 1 Allgemeine und mobilitätsbezogene Ressourcen stehen bei mobil Beschäftigten in positivem Zusammenhang mit Arbeitsfreude.

In Übereinstimmung mit den Annahmen des JD-R-Modells, liegt der Fokus der vorliegenden Studie auf der Bedeutung von Ressourcen für die Arbeitsfreude. Allerdings gibt es einige Studien, die zusätzlich einen negativen Zusammenhang zwischen Arbeitsbelastungen und motivationalen Prozessen nahelegen (u. a. Hakanen et al. 2008; Halbesleben 2010). Dabei zeigte sich in bisherigen Untersuchungen insbesondere die zeitliche Arbeitsbelastung in Form von Zeitdruck als bester Prädiktor. Hinzu kommen Reisedauer und Reisefrequenz (DeFrank et al. 2000; Ivancevich et al. 2003; Kraus und Rieder 2019). Deshalb werden in der vorliegenden Studie zusätzlich ausgewählte Belastungen einbezogen. Es wird Folgendes angenommen:

Hypothese 2 Belastungen stehen bei mobil Beschäftigen in negativem Zusammenhang mit Arbeitsfreude.

\section{Methoden}

\subsection{Stichprobe}

Um Faktoren zu untersuchen, die mit Arbeitsfreude assoziiert sind, wurden im Forschungsprojekt prentimo schriftliche Befragungen und Interviews mit mobil Beschäftigten durchgeführt. Vier Unternehmen der IT- sowie der Maschinenbaubranche nahmen teil..$^{1}$ Es wurden 407 Mitarbeitende, die regelmäßig Dienstreisen durchführen, in einer schriftlichen Onlineerhebung befragt. Zusätzlich erfolgten 59 qualitative Intensivinterviews. Entsprechend der Geschlechterverteilung in den beteiligten Branchen war die überwiegende Mehrheit der Befragten männlich (85\%). Durchschnittlich waren sie 43 Jahre alt $(S D=9)$ und seit acht Jahren im Unternehmen $(S D=6)$. Die Mehrheit $(93 \%)$ war in Vollzeit tätig, und $52 \%$ hatten Vertrauensarbeitszeit (48\% Gleitzeit). $20 \%$ hatten eine Führungsposition inne. Bei rund ein Drittel der Befragten (35\%) dauerten die Dienstreisen bis zu einen Tag, bei etwas

\footnotetext{
${ }^{1}$ Insgesamt wurden in prentimo mehr Unternehmen einbezogen, allerdings konnten nicht in allen Projektbetrieben Mitarbeiterbefragungen durchgeführt werden (zum Gesamtsample siehe Breisig und Vogl 2019).
} 
mehr als der Hälfte bis zu einer Woche und bei etwa einem Zehntel (11\%) länger als eine Woche.

\subsection{Erhebungs- und Auswertungsmethoden}

In Tab. 1 findet sich eine Übersicht der für die schriftliche Befragung eingesetzten Teilverfahren zur Untersuchung von Arbeitsbedingungen und Arbeitsfreude. Diese stammen teilweise aus bereits erprobten und publizierten Verfahren.

Die Skala Vielseitigkeit erfasst, inwieweit die Arbeit vielfältige Anforderungen stellt und die Möglichkeit bietet, individuelle Kompetenzen einzusetzen und zu erweitern. Mit dem Handlungsspielraum wird erfasst, inwieweit die Arbeit es ermöglicht, eigene Entscheidungen zur Planung und Einteilung der Arbeit zu treffen. Quantitative Belastungen beziehen sich auf eine zu hohe Arbeitsmenge und Zeitdruck in der Arbeit (Prümper et al. 1995). Mit der gekürzten Skala Führungsqua-

Tab. 1 Übersicht über die in der Befragung verwendeten Skalen und Einzelfragen

\begin{tabular}{|c|c|c|c|c|}
\hline Skalen & Fragen & $\begin{array}{l}\text { Reliabili- } \\
\text { tät }(\alpha)\end{array}$ & Beispielfrage & Instrumente \\
\hline \multicolumn{5}{|c|}{ Allgemeine Arbeitsbedingungen } \\
\hline Vielseitigkeit & 3 & 0,67 & $\begin{array}{l}\text { Bei meiner Arbeit habe ich insge- } \\
\text { samt gesehen häufig wechselnde, } \\
\text { unterschiedliche Arbeitsaufgaben }\end{array}$ & $\begin{array}{l}\text { KFZA (Prümper } \\
\text { et al. 1995) }\end{array}$ \\
\hline Handlungsspielraum & 3 & 0,78 & $\begin{array}{l}\text { Ich kann meine Arbeit selbststän- } \\
\text { dig planen und einteilen }\end{array}$ & \\
\hline $\begin{array}{l}\text { Quantitative Belas- } \\
\text { tungen }\end{array}$ & 2 & 0,66 & Ich stehe häufig unter Zeitdruck & \\
\hline Führungsqualität & 3 & 0,86 & $\begin{array}{l}\text { Meine unmittelbare Führungskraft } \\
\text { misst der Arbeitszufriedenheit } \\
\text { einen hohen Stellenwert bei }\end{array}$ & $\begin{array}{l}\text { COPSOQ (Nüb- } \\
\text { ling et al. 2005) }\end{array}$ \\
\hline \multicolumn{5}{|c|}{ Mobilitätsbezogene Arbeitsbedingungen } \\
\hline $\begin{array}{l}\text { Dauer der Einsätze } \\
\text { (Einzelfrage) }\end{array}$ & 1 & - & $\begin{array}{l}\text { Wie lange dauern Ihre Einsätze/ } \\
\text { Dienstreisen überwiegend? }\end{array}$ & $\begin{array}{l}\text { Amobil (Kraus } \\
\text { und Rieder 2018) }\end{array}$ \\
\hline $\begin{array}{l}\text { Häufigkeit der Ein- } \\
\text { sätze (Einzelfrage) }\end{array}$ & 1 & - & $\begin{array}{l}\text { Wie oft sind Sie pro Monat un- } \\
\text { gefähr auf Dienstreise oder an } \\
\text { verschiedenen Einsatzorten tätig? }\end{array}$ & \\
\hline $\begin{array}{l}\text { Mobilitätsbezogene } \\
\text { soziale Unterstüt- } \\
\text { zung }\end{array}$ & 2 & 0,69 & $\begin{array}{l}\text { Ich kann unterwegs bei meinen } \\
\text { mobilen Einsätzen anfallende } \\
\text { Fragen unkompliziert mit Kollegen } \\
\text { klären }\end{array}$ & \\
\hline $\begin{array}{l}\text { Mobilitätsbezogene } \\
\text { technische und orga- } \\
\text { nisatorische Unter- } \\
\text { stützung }\end{array}$ & 4 & 0,76 & $\begin{array}{l}\text { Wenn auf meinen mobilen Einsät- } \\
\text { zen Probleme mit mobiler Technik } \\
\text { entstehen, erhalte ich unkompli- } \\
\text { ziert Unterstützung }\end{array}$ & \\
\hline $\begin{array}{l}\text { Mobilitätsbezogener } \\
\text { Planungsspielraum }\end{array}$ & 3 & 0,89 & $\begin{array}{l}\text { Ich kann den Zeitpunkt der mobi- } \\
\text { len Einsätze selbst bestimmen }\end{array}$ & $\begin{array}{l}\text { Vogl et al. (2014) } \\
\text { sowie Amobil } \\
\text { (Kraus und Rieder } \\
\text { 2018) }\end{array}$ \\
\hline Arbeitsfreude & 3 & 0,87 & $\begin{array}{l}\text { Es gibt Tage, da freue ich mich } \\
\text { über meine Arbeit }\end{array}$ & $\begin{array}{l}\text { DIGA (Ducki } \\
2000)\end{array}$ \\
\hline
\end{tabular}


lität wird erfasst, inwieweit die Führungskraft aus Sicht der Mitarbeitenden gute Entwicklungsmöglichkeiten bietet, Konflikte gut löst und Wert auf die Mitarbeiterzufriedenheit legt (Nübling et al. 2005). Die Skala Arbeitsfreude wurde in gekürzter Fassung von Ducki (2000) übernommen. Sie erfasst positive Emotionen in der Arbeit, wobei der Fokus auf Freude und Stolz liegt.

Um speziell mobilitätsbezogene Arbeitsbedingungen zu untersuchen, wurde ein neues Instrument entwickelt, der Fragebogen zur Analyse mobiler Arbeit, Amobil (Kraus und Rieder 2018). Mit der mobilitätsbezogenen sozialen Unterstützung wird innerhalb dieses Verfahrens erfasst, inwieweit die Mitarbeitenden während ihrer Dienstreisen Zugang zu sozialer Unterstützung durch Kollegen und Vorgesetzte haben. Mobilitätsbezogene technische und organisatorische Unterstützung bezieht sich auf den Zugang zu erforderlichen Arbeitsmitteln, geeigneten Räumlichkeiten, Informationen sowie technischem Support unterwegs. Mit dem mobilitätsbezogenen Planungsspielraum wird erhoben, inwieweit die Mitarbeitenden über Fragen der Reisegestaltung mitentscheiden können. Diese Skala wurde von Vogl et al. (2014) entwickelt und in Amobil integriert. Die Rahmenbedingungen der Mobilität (Häufigkeit und Dauer) werden mit zwei Einzelfragen erfasst.

Die eingesetzten Skalen enthalten jeweils zwischen zwei und vier Fragen. Die Antwortmöglichkeiten sämtlicher Skalen umfassen fünf Stufen (von 1=, trifft über-

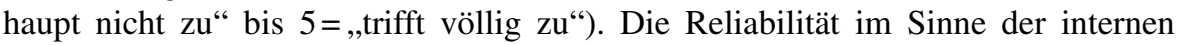
Konsistenz der Skalen liegt zwischen $\alpha=0,66$ und $\alpha=0,89$. Sämtliche Auswertungen der schriftlichen Befragung wurden mit der Statistiksoftware SPSS durchgeführt (IBM Corp. 2017).

Die leitfadengestützten Intensivinterviews mit mobil Beschäftigten dauerten jeweils zwischen 60 und 90 min. Im Mittelpunkt standen die differenzierte Erfassung der mobilitätsbezogenen Anforderungen, des Erlebens dieser Anforderungen und des individuellen Arbeitshandelns. Sämtliche Interviews wurden transkribiert. Die Auswertung erfolgte computergestützt (MAXQDA; Kuckartz 2018) auf Grundlage einer interpretativ und typisierend angelegten Methode. Die Auswertung des Materials erfolgte im Projektteam.

\section{Ergebnisse}

Die deskriptiven Ergebnisse zeigen, dass insbesondere Ressourcen in Form von Vielseitigkeit, Handlungsspielraum, mobilitätsbezogener technischer und organisatorischer Unterstützung sowie mobilitätsbezogener sozialer Unterstützung hoch ausgeprägt sind (Mittelwerte von mindestens 3,5 auf einer fünfstufigen Skala, s. Tab. 2). Quantitative Belastungen weisen einen Mittelwert von $M=3,29$ auf $(S D=0,92)$.

Die Ergebnisse zeigen außerdem, dass mobile Arbeit überwiegend mit viel Arbeitsfreude verbunden ist:

- Rund drei Viertel der mobil Beschäftigten (74\%) erleben hoch ausgeprägte Arbeitsfreude (Werte ab 3,5 auf einer fünfstufigen Skala).

- Rund ein Fünftel (21\%) liegt im mittleren Bereich (Werte zwischen 2,5 und 3,4).

- Nur wenige Befragte (5\%) geben geringe Arbeitsfreude an (Werte unter 2,5). 


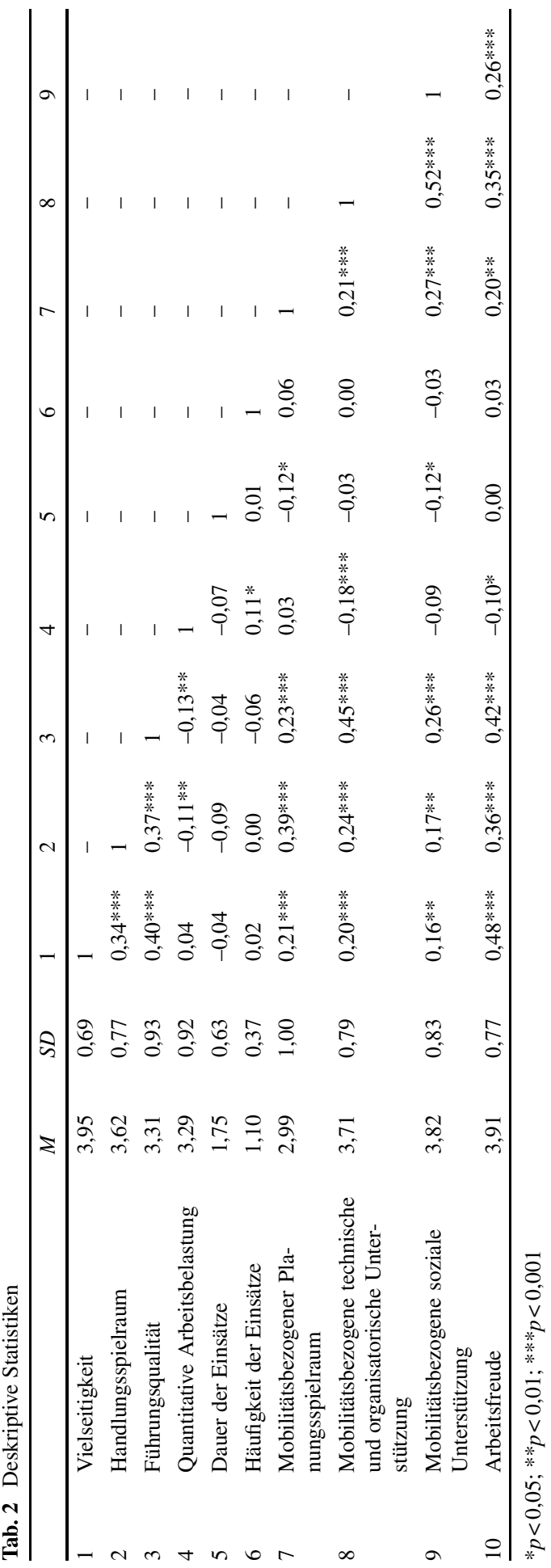


Tab. 3 Ergebnisse der hierarchischen Regressionsanalysen zur Erklärung von Arbeitsfreude $(n=407)$

\begin{tabular}{|c|c|c|c|}
\hline & \multicolumn{3}{|c|}{ Arbeitsfreude } \\
\hline & $\beta$ & $\beta$ & $\beta$ \\
\hline Geschlecht $^{\mathrm{a}}$ & $-0,09$ & $-0,08$ & $-0,09$ \\
\hline Alter & $-0,04$ & 0,05 & 0,02 \\
\hline Führungsverantwortung ${ }^{\mathrm{b}}$ & 0,11 & 0,02 & 0,03 \\
\hline Beschäftigungsumfang $^{\mathrm{c}}$ & $-0,07$ & $-0,07$ & $-0,09$ \\
\hline Arbeitszeitmodell $^{\mathrm{d}}$ & $-0,04$ & $-0,10$ & $-0,09$ \\
\hline Vielseitigkeit & - & $0,35 * * *$ & $0,34 * * *$ \\
\hline Handlungsspielraum & - & $0,18 * *$ & $0,15^{* *}$ \\
\hline Führungsqualität & - & $0,21 * * *$ & $0,14^{*}$ \\
\hline Quantitative Arbeitsbelastung & - & $-0,06$ & $-0,04$ \\
\hline Dauer der Einsätze & - & - & 0,04 \\
\hline Häufigkeit der Einsätze & - & - & 0,03 \\
\hline Mobilitätsbezogener Planungsspielraum & - & - & 0,01 \\
\hline Mobilitätsbezogene soziale Unterstützung & - & - & 0,09 \\
\hline $\begin{array}{l}\text { Mobilitätsbezogene technische und organisatorische Unter- } \\
\text { stützung }\end{array}$ & - & - & $0,15^{*}$ \\
\hline Korrigiertes $\mathrm{R}^{2}$ & 0,00 & 0,31 & 0,34 \\
\hline$\Delta \mathrm{R}^{2}$ & 0,00 & $0,31 * * *$ & $0,03 * *$ \\
\hline
\end{tabular}

$\beta$ standardisierter Regressionskoeffizient

${ }^{\mathrm{a}} 0=$ weiblich, $1=$ männlich

${ }^{\mathrm{b}} 0=$ nein, $1=\mathrm{ja}$

${ }^{\mathrm{c}} 0=$ Vollzeit, $1=$ Teilzeit

$\mathrm{d}_{0}=$ Gleitzeit, $1=$ Vertrauensarbeitszeit

$* p<0,05 ; * * p<0,01 ; * * * p<0,001$

Der Durchschnitt liegt bei einem Wert von 3,91 ( $S D=0,77$; s. Tab. 2). Arbeitsfreude wird auch von mobil Beschäftigten in den Interviews erwähnt. So formuliert ein IT-Berater:

Also ich empfinde es in Summe nicht als belastend. Im Gegenteil, ich habe da tatsächlich Spaß dran.

Zur Überprüfung der Hypothesen wurden Zusammenhänge zwischen Arbeitsbedingungen und Arbeitsfreude ausgewertet. Dies erfolgte mittels einer multiplen hierarchischen Regression (s. Tab. 3). Dabei werden schrittweise mögliche Einflussfaktoren auf Arbeitsfreude in die Berechnung einbezogen. Es wurde geprüft, welchen Beitrag diese jeweils zum Entstehen der Arbeitsfreude leisten.

Im ersten Schritt wurden demografische Merkmale (Alter und Geschlecht) sowie Merkmale der Beschäftigungssituation (Führungsverantwortung, Beschäftigungsumfang sowie Arbeitszeitmodell) einbezogen. Die standardisierten Regressionskoeffizienten $\beta$ sind jeweils niedrig und statistisch nicht signifikant. Ob jemand weiblich oder männlich ist, alt oder jung, Führungsverantwortung trägt oder nicht, ist somit nicht wichtig für die Freude an der mobilen Tätigkeit. Dementsprechend ist die Varianzaufklärung des Modells in diesem ersten Schritt Null (korrigiertes $\mathrm{R}^{2}=0,00$ ).

Im zweiten Schritt wurden allgemeine Arbeitsbedingungen in die Berechnung eingefügt. Hier zeigt sich, dass eine hohe Vielseitigkeit der Arbeit $(\beta=0,35 ; p<0,001)$, 
ein hoher Handlungsspielraum $(\beta=0,18 ; p<0,01)$ sowie eine gute Führungsqualität $(\beta=0,21 ; p<0,001)$ signifikant zur Arbeitsfreude beitragen. Auch in den Interviews weisen die mobil Beschäftigten auf die Vorteile hoher Handlungsspielräume hin. Ein Servicetechniker drückt dies folgendermaßen aus:

Ich bin relativ flexibel in dem, was ich tue. Ich habe keinen, der mir sagt, was ich machen muss und wie ich es machen muss, wann ich es machen muss. Es gibt ein Zeitfenster, in dem ich das erledigt haben muss, aber ich habe keinen Chef.

Die engsten Zusammenhänge bestehen zwischen Vielseitigkeit der Arbeit und Arbeitsfreude $(\beta=0,35 ; p<0,001)$. Ein IT-Berater, der bedingt durch häufige Kundenbesuche beruflich viel unterwegs ist, formuliert dazu:

Ich liebe diese Abwechslung, heute hier zu sein, morgen da zu sein, verschiedenste Menschen zu treffen, verschiedene Städte, auch wenn auch manchmal nur vom Durchfahren zu sehen. Das ist für mich ein ganz wesentlicher Aspekt. Ich bin kein klassischer Nine-to-Five-Mensch, der morgens sich an seinen Schreibtisch setzt, seine Arbeit macht und um fünf wieder nach Hause geht.

Quantitative Arbeitsbelastungen hängen demgegenüber nicht mit Arbeitsfreude zusammen. Arbeitsfreude kann somit bei Tätigkeiten mit hohem und niedrigem Zeitdruck gleichermaßen auftreten.

Die allgemeinen Arbeitsbedingungen tragen gemeinsam deutlich zur Varianzaufklärung bei, diese beträgt nun $31 \%$ (korrigiertes $\mathrm{R}^{2}=0,31$ ). Das bedeutet, dass Unterschiede der Arbeitsfreude zu fast einem Drittel durch die allgemeinen Arbeitsbedingungen erklärt werden können.

Im dritten Schritt wurde geprüft, ob mobilitätsbezogene Arbeitsbedingungen zusätzlich zum Entstehen von Arbeitsfreude beitragen. Hier zeigen sich überwiegend geringe Zusammenhänge. Eine Ausnahme ist die mobilitätsbezogene technische und organisatorische Unterstützung $(\beta=0,15 ; p<0,05)$. Diese erweist sich als bedeutsame Ressource für Arbeitsfreude. Wer unterwegs eine bessere Unterstützung erfährt, erlebt statistisch signifikant mehr Arbeitsfreude.

Die Bedeutung technischer und organisatorischer Unterstützung unterwegs geht auch aus den Interviews mit mobil Beschäftigten hervor. Fehlen z. B. wichtige Informationen, die zur Bearbeitung des Arbeitsauftrags benötigt werden, kann dies zu Belastungssituationen führen:

Man sitzt im Auto, man ist relativ handlungsunfähig oder weiß, man ist in zehn Minuten beim Kunden im Termin, wo man eigentlich die Firma und die Produkte an den Mann bringen muss. Wo man überzeugend auftreten muss und hinter der Sache stehen muss, dass wir genau der richtige Partner sind, und gerade vorher telefoniert man sich die Finger wund, weil bei einem anderen Kunden irgendein Problem aufgetreten ist und keiner sich zuständig fühlt, keiner sich drum kümmern will. Und das sind echte Stressmomente.

Mit dem Einbezug der mobilitätsbezogenen Arbeitsbedingungen wird die Varianzaufklärung weiter verbessert und liegt nun bei $34 \%$ (korrigiertes $\mathrm{R}^{2}=0,34$ ). Die 
Verbesserung der Varianzaufklärung gegenüber dem zweiten Schritt liegt somit bei $3 \%$ und ist statistisch signifikant $\left(\Delta \mathrm{R}^{2}=0,03\right)$.

\section{Diskussion}

In der vorliegenden Studie wurde geprüft, welchen Beitrag arbeitsbezogene Ressourcen bei berufsbedingter Mobilität für die Arbeitsfreude - als Indikator für intrinsische Motivation - der Beschäftigten leisten. Es wurden allgemeine Ressourcen wie Handlungsspielraum, Vielseitigkeit und Führungsqualität sowie mobilitätsbezogene Ressourcen wie mobilitätsbezogene soziale sowie technisch-organisatorische Unterstützung untersucht. Dabei lehnt sich die Studie an theoretische Überlegungen von Bakker und Demerouti $(2008,2014,2017)$ an.

Sowohl aus den qualitativen Interviews als auch aus den quantitativen Daten geht hervor, dass mobile Arbeit mehrheitlich positiv wahrgenommen wird und im Allgemeinen mit Arbeitsfreude assoziiert ist. Dieses Ergebnis ist überraschend, wenn man berücksichtigt, dass mobile Arbeit vielfach hohe Belastungen aufweist (Kraus und Rieder 2019; Nies et al. 2017). Eine Erklärung bieten die Ergebnisse der hierarchischen multiplen Regression. Sie zeigen, dass allgemeine Arbeitsressourcen in Form von Handlungsspielraum, Vielseitigkeit der Arbeitstätigkeit und Führungsqualität zum Entstehen von Arbeitsfreude beitragen. Darüber hinaus hängen mobilitätsbezogene Ressourcen in Form von mobilitätsbezogener technischer und organisatorischer Unterstützung positiv mit Arbeitsfreude zusammen. Die Ergebnisse bestätigen somit die erste Hypothese. Sie stehen außerdem in Übereinstimmung mit dem JobDemands-Resources Model (Bakker und Demerouti 2014, 2017).

Zur Überprüfung der zweiten Hypothese wurde der Zusammenhang zwischen Arbeitsbelastungen, Umfang der Reisen und Arbeitsfreude untersucht. Weder Zeitdruck noch Reisedauer oder Reisehäufigkeit zeigten einen Zusammenhang mit Arbeitsfreude, so dass die zweite Hypothese nicht bestätigt wurde. Zugleich sind diese Ergebnisse ebenfalls übereinstimmend dem JD-R-Modell, dementsprechend Belastungen (demands) keinen direkten Einfluss auf die Mitarbeitermotivation haben.

\subsection{Limitationen}

Insbesondere das querschnittliche Design der Studie trägt zu Limitationen bei, weshalb keine kausalen Zusammenhänge zwischen Arbeitsbedingungen und Arbeitsfreude geprüft werden können. Weiterhin blieben mögliche Einflussfaktoren auf Arbeitsfreude jenseits von Arbeitsbedingungen unberücksichtigt. So ist davon auszugehen, dass auch Persönlichkeitsmerkmale sowie soziale Kontextfaktoren (z.B. die Familiensituation) hierfür bedeutsam sind. Es ist außerdem möglich, dass durch die Selbstberichte der Beschäftigten, in denen alle Variablen in einer einzigen schriftlichen Befragung erfasst wurden, eine gemeinsame Methodenvarianz entstanden ist, welche die Ergebnisse beeinflusst. Allerdings sprechen auch die Ergebnisse aus den qualitativen Interviews für die vorliegenden Effekte. Eine weitere Limitation ergibt sich aus der vorliegenden Stichprobe. Sie ist nicht repräsentativ für berufsbedingte 
Mobilität, da es sich speziell um Unternehmen aus der IT- und Maschinenbaubranche handelt.

\subsection{Praktische Implikationen}

Starre Strukturen und langwierige Prozesse dominieren, gerade in großen deutschen Unternehmen, den Arbeitsalltag. Die Unternehmenskultur hat sich vielfach noch nicht den Anforderungen einer flexibler, mobiler und komplexer werdenden Arbeitswelt angepasst. Eine Herausforderung für Unternehmen und Führungskräfte ist dementsprechend die Motivation, Bindung und Inspiration der Beschäftigten. Die vorliegende Studie zeigt, dass es hierfür zentrale Ressourcen gibt, die in Unternehmen, auch bei berufsbedingter Mobilität, gestärkt werden können. Hierzu gehören der Handlungsspielraum sowie die Vielseitigkeit der Tätigkeit. Arbeitsfreude steht außerdem mit Führungsqualität in Zusammenhang. Dementsprechend sollten Führungskräfte von mobilen Mitarbeitenden zu den Besonderheiten der Führung bei mobiler Arbeit geschult werden.

Den Führungskräften kommt aber auch eine weitere Aufgabe zu: Im Sinne des Job Crafting (Wrzesniewski und Dutton 2001) können Beschäftigte den nötigen Spielraum erhalten, um bestimmte Belange der Arbeitstätigkeit selbst den eigenen Bedürfnissen anzupassen. Beispielsweise kann es für mobil Beschäftigte, die überwiegend allein unterwegs sind, enorm hilfreich sein, soziale Kontakte zum heimischen Unternehmen aufrecht zu erhalten. Dies kann sowohl digital als auch analog passieren. Nicht selten werden dazu informelle Strategien - z.B. Gruppen über private Messengerdienste - gewählt, damit ein Austausch zwischen den Kollegen stattfinden kann. Tims et al. (2013) zeigen in ihrer Studie, dass Beschäftigte, die Job Crafting anwenden, ihre Ressourcen über einen Zweimonatszeitraum steigern konnten. Demnach ist eine wichtige Stellschraube in der praktischen Arbeit die Befähigung der Beschäftigten, die eigenen Arbeitsbedingungen zu beeinflussen (s. auch Empowerment; Seibert et al. 2011). Im Forschungsprojekt prentimo wurde dies unter anderem über sogenannte Mobilitätswerkstätten realisiert (Peters 2017). In diesen können mobile Mitarbeitende und Führungskräfte partizipativ die Arbeitsbedingungen erfassen und gestalten. Auch ein individuelles Coaching zum Erwerb spezifischer Mobilitätskompetenzen kann wirkungsvoll sein (Nies et al. 2015).

Eine Grundlage all dieser Personalentwicklungsinstrumente kann eine Gefährdungsbeurteilung psychischer Belastungen sein, die neben den Belastungen auch die Ressourcen der mobilen Tätigkeit im Unternehmen erfasst. Um neben allgemeinen Arbeitsbedingungen auch mobilitätsbezogene Aspekte zu integrieren, kann das Instrument Amobil (Kraus und Rieder 2018) verwendet werden.

\section{Schlussbemerkung}

Mobile Arbeit bietet vielfältige Möglichkeiten: Neben einem kulturell und gesellschaftlich bildenden Charakter sind mobile Tätigkeiten häufig abwechslungsreich und ermöglichen viele selbstständige Entscheidungen der Beschäftigten. Mit Ressourcen wie Handlungsspielraum, Vielseitigkeit oder mobilitätsbezogener techni- 
scher und organisatorischer Unterstützung geht bei mobiler Arbeit eine höhere Arbeitsfreude einher. Essenziell für die betriebliche Praxis ist daher eine Förderung dieser Ressourcen. Auf diese Weise können die Chancen, die für die Mitarbeitenden und die Unternehmen in den Besonderheiten mobiler Arbeit liegen, gezielt genutzt werden.

Förderung Das Projekt Präventionsorientierte Gestaltung mobiler Arbeit - prentimo wurde durch das Bundesministerium für Bildung und Forschung im Rahmen des Programms „Arbeiten - Lernen - Kompetenzen entwickeln. Innovationsfähigkeit in einer modernen Arbeitswelt“ im Förderschwerpunkt „Präventive Maßnahmen für die sichere und gesunde Arbeit von morgen“ gefördert. Laufzeit des Projektes: 01.01.2016-31.07.2019. Nähere Informationen dazu finden sich unter: www.prentimo.de.

Funding Open Access funding provided by Projekt DEAL.

Open Access Dieser Artikel wird unter der Creative Commons Namensnennung 4.0 International Lizenz veröffentlicht, welche die Nutzung, Vervielfältigung, Bearbeitung, Verbreitung und Wiedergabe in jeglichem Medium und Format erlaubt, sofern Sie den/die ursprünglichen Autor(en) und die Quelle ordnungsgemäß nennen, einen Link zur Creative Commons Lizenz beifügen und angeben, ob Änderungen vorgenommen wurden.

Die in diesem Artikel enthaltenen Bilder und sonstiges Drittmaterial unterliegen ebenfalls der genannten Creative Commons Lizenz, sofern sich aus der Abbildungslegende nichts anderes ergibt. Sofern das betreffende Material nicht unter der genannten Creative Commons Lizenz steht und die betreffende Handlung nicht nach gesetzlichen Vorschriften erlaubt ist, ist für die oben aufgeführten Weiterverwendungen des Materials die Einwilligung des jeweiligen Rechteinhabers einzuholen.

Weitere Details zur Lizenz entnehmen Sie bitte der Lizenzinformation auf http://creativecommons.org/ licenses/by/4.0/deed.de.

\section{Literatur}

Bakker, A. B., \& Demerouti, E. (2008). Towards a model of work engagement. Career Development International, 13(3), 209-223. https://doi.org/10.1108/13620430810870476.

Bakker, A. B., \& Demerouti, E. (2014). Job demands-resources theory. In C. Cooper \& P. Chen (Hrsg.), Wellbeing: a complete reference guide (S. 37-64). Chichester: Wiley-Blackwell.

Bakker, A. B., \& Demerouti, E. (2017). Job demands-resources theory: taking stock and looking forward. Journal of Occupational Health Psychology, 22, 273-285. https://doi.org/10.1037/ocp0000056.

Bornheim-Gallemeister, N. (2013). Positive Emotionen in der Arbeitswelt. Eine vergleichende Untersuchung über das Erleben von Rahmenbedingungen und Arbeitserleben in der stationören Altenpflege (Unveröffentlichte Dissertation). Bremen: Universität Bremen.

Breisig, T., \& Vogl, G. (2019). Mobile Arbeit gesund gestalten - ein Praxishandbuch. Oldenburg: Carl von Ossietzky Universität. http://www.prentimo.de/assets/Uploads/prentimo-Broschuere-Screen.pdf

DeFrank, R. S., Konopaske, R., \& Ivancevich, J.M. (2000). Executive travel stress: perils of the road warrior. Academy of Management Executive, 14, 28-71.

Ducki, A. (2000). Diagnose gesundheitsförderlicher Arbeit: Eine Gesamtstrategie zur betrieblichen Gesundheitsanalyse. Zürich: vdf Hochschulverlag.

Ducki, A., \& Nguyen, H. T. (2016). Psychische Gesundheit in der Arbeitswelt - Mobilität. Dortmund: Bundesanstalt für Arbeitsschutz und Arbeitsmedizin. https://doi.org/10.21934/baua:bericht20160713/3d.

Espino, C. M., Sundstrom, S.M., Frick, H.L., Jacobs, M., \& Peters, M. (2002). International business travel: Impact on families and travellers. Occupational and Environmental Medicine, 59, 309-322. https://doi.org/10.1136/oem.59.5.309.

Fried, Y., \& Ferris, G. R. (1987). The validity of the job characteristics model: a review and meta-analysis. Personnel Psychology, 40(2), 287-322.

Gagné, M., \& Deci, E. L. (2005). Self-determination theory and work motivation. Journal of Organizational Behavior, 26(4), 331-362. https://doi.org/10.1002/job.322.

Hackman, J. R., \& Oldham, G. R. (1980). Work redesign. Reading: Addison-Wesley. 
Hakanen, J., Schaufeli, W.B., \& Ahola, K. (2008). The job demands-resources model: a three-year crosslagged study of burnout, depression, commitment and work engagement. Work \& Stress, 22, 224-241. https://doi.org/10.1080/02678370802379432.

Halbesleben, J. (2010). A meta-analysis of work engagement: relationships with burnout, demands, resources, and consequences. In A. Bakker \& M. Leiter (Hrsg.), Work engagement-A handbook of essential theory and research (S. 102-111). New York: Psychol. Press.

Hupfeld, J., Brodersen, S., \& Herdegen, R. (2013). Arbeitsbedingte räumliche Mobilität und Gesundheit. IGA-Report 25. Verfügbar unter https://www.iga-info.de/veroeffentlichungen/igareporte/igareport25/. Zugegriffen: 16. Jan. 2019.

IBM Corp (2017). IBM SPSS Statistics for Windows, Version 25.0. Armonk: IBM Corp.

Ivancevich, J. M., Konopaske, R., \& DeFrank, R.S. (2003). Business travel stress: a model, propositions and managerial implications. Work \& Stress, 17, 138-157.

Jensen, M. T. (2014). Exploring business travel with work-family conflict and the emotional exhaustion component of burnout as outcome variables: The job demands-resources perspective. European Journal of Work and Organizational Psychology, 23, 497-510. https://doi.org/10.1080/1359432X. 2013.787183.

Kesselring, S., \& Vogl, G. (2010). Betriebliche Mobilitätsregime. Berlin: edition sigma.

Kraus, S., \& Rieder, K. (2019). Mobilitätsbezogene Arbeitsbedingungen und interessierte Selbstgefährdung: Bedeutung für die psychische Gesundheit. Wirtschaftspsychologie, 21(1/2), 6-16.

Kraus, S., \& Rieder, K. (2018). Fragebogen zur Analyse mobiler Arbeit - Amobil. Aalen: Hochschule Aalen. https://www.prentimo.de/fragebogen-amobil. Zugegriffen: 16. Jan. 2019.

Kuckartz, U. (2018). Qualitative Inhaltsanalyse. Methoden, Praxis, Computerunterstützung (4. Aufl.). Weinheim, Basel: Beltz Juventa.

Lüdemann, P. M. (2016). Gesundheit und Gesundheitsmanagement im selbstständigen Außendienst. Unveröff. Dissertation. Universität Bielefeld.

Mäkelä, L., \& Kinnunen, U. (2016). International business travelers' psychological well-being: The role of supportive HR practices. The International Journal of Human Resource Management, 29(7), 1285-1306. https://doi.org/10.1080/09585192.2016.1194872.

Meller, S., \& Ducki, A. (2002). Tätigkeitsbezogene Begeisterung in der Erwerbsarbeit. Arbeit, 2(11), 101-116.

Müller, T., Hommelhoff, S., Westman, M., \& Niessen, C. (2017). Control and meaning in the context of business travel. Presentation at the 77th Annual Academy of Management Conference, Atlanta.

Nicholas, H., \& McDowall, A. (2012). When work keeps us apart: a thematic analysis of the experience of business travellers. Community, Work and Family, 15, 335-355. https://doi.org/10.1080/13668803. 2012.668346.

Nies, S., Roller, K., \& Vogl, G. (2015). Räumliche Mobilität rund um die Arbeit. Düsseldorf: Hans-Böckler-Stiftung.

Nies, S., Roller, K., \& Vogl, G. (2017). Mobilität und Leistung: Dienstreisende im Trade-Off zwischen Verdichtung und Entgrenzung. Arbeit, 26(2), 173-191. https://doi.org/10.1515/arbeit-2017-0011.

Niessen, C., Müller, T., Hommelhoff, S., \& Westman, M. (2018). The impact of preventive coping on business travelers' work and private life. Journal of Organizational Behavior, 39(1), 113-127. https:// doi.org/10.1002/job.2215.

Nübling, M., Stößel, U., Hasselhorn, H. M., Michaelis, M., \& Hofmann, F. (2005). Methoden zur Erfassung psychischer Belastungen. Erprobung eines Messinstrumentes (COPSOQ). Dortmund: Bundesanstalt für Arbeitsschutz und Arbeitsmedizin.

Pangert, B., Pauls, N., Schlett, C., \& Menz, W. (2017). Ständige Erreichbarkeit - Ursachen, Auswirkungen, Gestaltungsansätze. http://erreichbarkeit.eu/images/Ergebnisbroschuere_250817.pdf. Zugegriffen: 16. Jan. 2019.

Peters, K. (2017). Interessierte Selbstgefährdung, indirekte Steuerung und mobile Arbeit. In T. Breisig, H. Grzech-Sukalo \& G. Vogl (Hrsg.), Mobile Arbeit gesund gestalten: Trendergebnisse aus dem Forschungsprojekt prenitmo - präventionsorientierte Gestaltung mobiler Arbeit (S. 12-14). Zugriff am 14.10.2019. Verfügbar unter https://www.prentimo.de/assets/Uploads/prentimo-Mobile-Arbeitgesund-gestalten.pdf.

Prümper, J., Hartmannsgruber, K., \& Frese, M. (1995). KFZA. Kurz-Fragebogen zur Arbeitsanalyse. Zeitschrift für Arbeits- und Organisationspsychologie, 39(3), 125-131.

Rau, R., \& Göllner, M. (2019). Erreichbarkeit gestalten, oder doch besser die Arbeit? Zeitschrift für Arbeits- und Organisationspsychologie, 63(1), 1-14. https://doi.org/10.1026/0932-4089/a000284.

Roth, I. (2017). Digitalisierung und Arbeitsqualität. Eine Sonderauswertung auf Basis des DGB-Index Gute Arbeit 2016 für den Dienstleistungssektor. Berlin: ver.di. unter Mitarbeit von N. Müller 
Seibert, S.E., Wang, G., \& Courtright, S.H. (2011). Antecedents and consequences of psychological and team empowerment in organizations: a meta-analytic review. Journal of Applied Psychology, 96(5), 981-1003.

Tims, M., Bakker, A. B., \& Derks, D. (2013). The impact of job crafting on job demands, job resources, and well-being. Journal of Occupational Health Psychology, 18(2), 230-240. https://doi.org/10.1037/ a0032141.

Ulich, E., \& Wülser, M. (2018). Gesundheitsmanagement in Unternehmen (7. Aufl.). Wiesbaden: Gabler.

VDR - Verband deutsches Reisemanagement (2019). VDR-Geschäftsreiseanalyse 2019. https://www. vdr-service.de/fileadmin/services-leistungen/fachmedien/geschaeftsreiseanalyse/VDRGeschaeftsreiseanalyse-2019.pdf. Zugegriffen: 20. Sept. 2019.

Vogl, G., Roller, K., Eichmann, V., Schiml, N., \& Pangert, B. (2014). Mobilität ,,rund um die Arbeit": Ergebnisse einer quantitativen Befragung. www.prentimo.de/assets/Uploads/Vogl-u.a.-2014Broschure-Mobilitat-rund-um-die-Arbeit-Ergebnisse-quanti-Befragung.pdf. Zugegriffen: 2. Mai 2019.

Westman, M., \& Etzion, D. (2002). The impact of short overseas business trips on job stress and burnout. Applied Psychology, 51(4), 582-592.

Westman, M., Etzion, D., \& Chen, S. (2009). Crossover of positive experiences from business travelers to their spouses. Journal of Managerial Psychology, 24, 269-284. https://doi.org/10.1108/ 02683940910939340.

Wrzesniewski, A., \& Dutton, J.E. (2001). Crafting a job: Revisioning employees as active crafters of their work. Academy of Management Review, 26(2), 179-201.



Prof. Dr. Kerstin Rieder Studium der Psychologie mit dem Schwerpunkt Arbeits- und Organisationspsychologie sowie Promotion an der TU Berlin. Arbeitsschwerpunkte: mobile Arbeit, betriebliches Gesundheitsmanagement, interaktive Arbeit, arbeitender Kunde. An der Hochschule Aalen Leitung des Studiengangs Wirtschaftspsychologie, Forschungsprojekt ,prentimo - präventionsorientierte Gestaltung mobiler Arbeit"(2016-2019). 


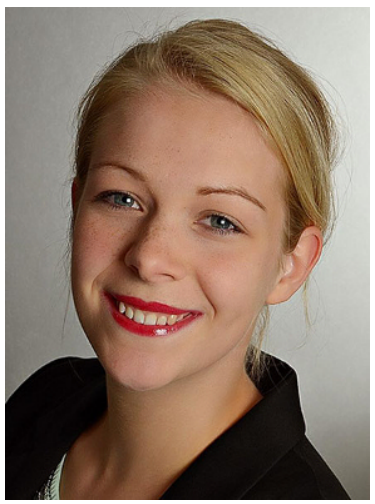

Sylvia Kraus Studium der Psychologie an den Universitäten in Magdeburg und Jena mit Vertiefung Arbeits- und Organisationspsychologie. Von 2016 bis 2019 wissenschaftliche Mitarbeiterin und Doktorandin an der Hochschule Aalen im Forschungsprojekt ,prentimo - präventionsorientierte Gestaltung mobiler Arbeit." Forschungsinteressen: mobile Arbeit, digitalisierte und agile Arbeitsformen sowie der Persönlichkeitsforschung.

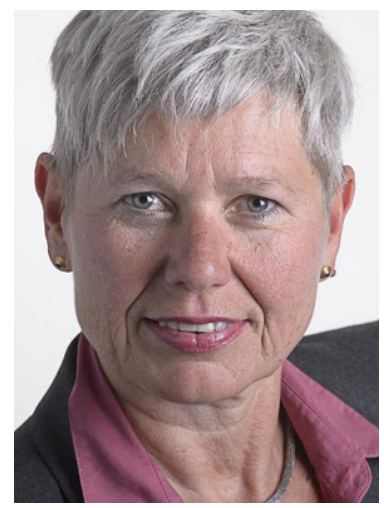

Dr. Gerlinde Vogl Studium der Soziologie (Schwerpunkt Arbeitsund Techniksoziologie, Arbeitspsychologie und Recht für Sozialwissenschaftler) in München und Wien, Promotion an der TU München. Arbeitsschwerpunkte: mobile Arbeit sowie neue Selbstständigkeit. 2016-2019 Projektleiterin des Forschungsprojekts ,prentimo - präventionsorientierte Gestaltung mobiler Arbeit“" (www.prentimo.de). 